This document is the accepted manuscript version of the following article: Kubacka, T., Johnson, J. A., Hoffmann, M. C., Vicario, C., de Jong, S., Beaud, P., ... Staub, U. (2014). Large-amplitude spin dynamics driven by a THz pulse in resonance with an electromagnon. Science, 343(6177), 1333-1336.

https://doi.org/10.1126/science. 1242862

This is the author's version of the work. It is posted here by permission of the AAAS for personal use, not for redistribution. The definitive version was published in Science 343, 1333 on March 21, 2014, DOI: 10.1126/science.1242862 .

\title{
Large amplitude spin dynamics driven by a THz pulse in resonance with an
}

\section{electromagnon}

Authors: T. Kubacka ${ }^{*}$, J.A. Johnson ${ }^{2}$, M.C. Hoffmann ${ }^{3}$, C. Vicario ${ }^{4}$, S. de Jong ${ }^{3}$, P. Beaud ${ }^{2}$, S. Grübel $^{2}$, S-W. Huang ${ }^{2}$, L. Huber ${ }^{1}$, L. Patthey ${ }^{4}$, Y-D. Chuang ${ }^{5}$, J.J. Turner ${ }^{3}$, G.L. Dakovski ${ }^{3}$, W-S. Lee $^{3}$, M.P. Minitti ${ }^{3}$, W. Schlotter ${ }^{3}$, R.G. Moore ${ }^{6}$, C.P. Hauri ${ }^{4,7}$, S.M. Koohpayeh ${ }^{8}$, V. Scagnoli ${ }^{2}$, G. Ingold ${ }^{2}$, S.L. Johnson ${ }^{1}$, U. Staub ${ }^{2}$

\section{Affiliations:}

${ }^{1}$ ETH Zurich, Institute for Quantum Electronics, Wolfgang-Pauli-Strasse 16, 8093 Zurich,

Switzerland

${ }^{2}$ Swiss Light Source, Paul Scherrer Institut, 5232 Villigen PSI, Switzerland

${ }^{3}$ Linac Coherent Light Source, SLAC National Accelerator Laboratory, Menlo Park, California 94025, USA

${ }^{4}$ SwissFEL, Paul Scherrer Institut, 5232 Villigen PSI, Switzerland

${ }^{5}$ Advanced Light Source, Lawrence Berkeley National Laboratory, Berkeley, California 94720, USA

${ }^{6}$ The Stanford Institute for Materials and Energy Sciences (SIMES), SLAC National Accelerator Laboratory, Menlo Park, California 94025, USA

${ }^{7}$ Ecole Polytechnique Federale de Lausanne, 1015 Lausanne, Switzerland 
${ }^{8}$ Institute for Quantum Matter, Department of Physics and Astronomy, Johns Hopkins University, Baltimore, MD 21218, USA

*Correspondence to: tkubacka@phys.ethz.ch

Abstract: Multiferroics have attracted strong interest for potential applications where electric fields control magnetic order. The ultimate speed of control via magnetoelectric coupling, however, remains largely unexplored. Here we report on an experiment in which we drive spin dynamics in multiferroic $\mathrm{TbMnO}_{3}$ with an intense few-cycle terahertz (THz) light pulse tuned to resonance with an electromagnon, an electric-dipole active spin excitation. We observe the resulting spin motion using time-resolved resonant soft x-ray diffraction. Our results show that it is possible to directly manipulate atomic-scale magnetic structures using the electric field of light on a sub-picosecond timescale.

Main Text: Data storage devices based on ferromagnetic or ferroelectric materials depend strongly on domain reorientation, a process that typically occurs over time scales of several nanoseconds. Faster reorientation dynamics may be achievable using intense electromagnetic (EM) pulses (1). The EM pulses can couple to magnetism either indirectly via electronic excitations (2) or directly via the Zeeman torque induced by the magnetic field (3-5). Direct excitation has the advantage of minimal excess heat deposition, but requires frequencies in the $10^{10}-10^{12} \mathrm{~Hz}$ range. The low magnetic field strength of currently realizable THz frequency EM sources poses a formidable challenge for such schemes.

Thanks to the coexistence of different ferroic orders, multiferroics offer new routes to domain control (6). Particularly strong coupling between the ferroelectric and magnetic order exists in single-phase frustrated magnets where noncollinear spin structure drives ferroelectricity as a result of weak relativistic interactions (7-9). Consequently, the magnetic order can be 
controlled by application of an electric field (10-13). The speed of domain switching triggered by simple step-function-like electric fields appears, however, to be limited to a timescale of several milliseconds (14). As an alternate solution, optical pulses have been shown to affect the magnetic structure of multiferroics on a femto- and picosecond timescale (15-17). It has been predicted that ultrafast magnetic dynamics can be also triggered by coherent excitation of electromagnons, electric-dipole active spin excitations directly connected to the magnetoelectric coupling (18). Here we show experimentally that a few-cycle THz pulse tuned to resonance with an electromagnon can transiently modify the magnetic structure of multiferroic $\mathrm{TbMnO}_{3}$.

$\mathrm{TbMnO}_{3}$ is a model spin-cycloid multiferroic exhibiting strong magnetoelectric coupling. Although it has a relatively simple perovskite atomic structure, a strong $\mathrm{GdFeO}_{3}$-type distortion gives rise to a variety of spin-frustrated phases $(19,20)$. At room temperature, the crystal is paramagnetic. Below $42 \mathrm{~K}$ the Mn spins form a paraelectric sinusoidally modulated spin density wave (SDW) state which transforms into a spin-cycloid state below $27 \mathrm{~K}$. In this phase the spins form a cycloid within the $(b c)$ crystallographic plane (Fig. 1A) and a spontaneous ferroelectric polarization along the $c$ axis develops. Microscopically, the spin current between canted spins on neighboring sites $i, j$ gives rise to a ferroelectric polarization $p_{i j} \propto r_{i j} \times\left(S_{i} \times S_{j}\right)(7)$, and the magnitude of the polarization is further enhanced by lattice displacements (21-23). In all these spin frustrated phases the magnetic structure of the Mn spins is incommensurate with the lattice, characterized by a wave vector $\mathbf{k}=(0, q, 0)$, where $q \approx 0.28$ changes very slowly in the SDW phase with temperature (24).

The EM excitation spectrum of $\mathrm{TbMnO}_{3}$ shows broad peaks in the $\mathrm{THz}$ frequency range; these have been assigned to electromagnons (25-29) (Fig. 1B, lower inset). The strongest feature at $1.8 \mathrm{THz}$ is activated with the electric component of light parallel to the $a$ axis and is absent in 
other geometries (26). It has been proposed that the oscillating electric field along the $a$ axis modifies the nearest neighbor ferromagnetic exchange constant in the $(a b)$ plane, resulting in anti-phase spin oscillations within the spin cycloid plane $(28,30)$. Weaker spectral features at lower frequencies have been proposed to arise from the higher harmonics of the spin cycloid and coupling to the strongest electromagnon (30), and from out-of-plane spin cycloid motions $(28$, $31,32)$

To investigate whether excitation of electromagnons in $\mathrm{TbMnO}_{3}$ is a viable route for magnetic order control, we performed a THz pump and soft x-ray probe experiment (Fig. 1B). The sample is a single crystal of $\mathrm{TbMnO}_{3}$ cut to the (010) surface, oriented so that the $a$ axis is at $45^{\circ}$ with respect to the horizontal scattering plane. We generated few-cycle, phase stable $\mathrm{THz}$ pulses with a center frequency of $1.8 \mathrm{THz}$ using optical rectification in a nonlinear organic crystal with a peak electric field of approximately $300 \mathrm{kV} / \mathrm{cm}$ at focus (33). We measured the electric field component of the $\mathrm{THz}$ waveform at the sample position using electro-optical sampling. To see the spin motion resulting from the excitation we used time-resolved resonant soft X-ray diffraction at the $\mathrm{Mn} L_{2}$ edge and measured the intensity of the first order $(0 q 0)$ cycloid reflection (34).

The spin dynamics can be extracted from the behavior of the intensity of the $(0 q 0)$ diffraction peak as a function of pump-probe delay time $\Delta \tau$ (Fig. 2). At $T=13 \mathrm{~K}$, where $\mathrm{TbMnO}_{3}$ is deep in the multiferroic phase, the $\mathrm{x}$-ray signal shows oscillations resembling the shape of the THz pump pulse electric field (Fig. 2A). The observed modulation of the diffraction peak intensity is over an order of magnitude larger than expected for unconstrained spin precession driven directly by the magnetic field component of the THz pulse (34). The Fourier transform of the x-ray trace (Fig. 2D) shows that the material response has essentially the same 
frequency spectrum as both the pump and the electromagnon. The delay between the first maximum of the pump trace and the first maximum of the x-ray trace is $250 \mathrm{fs}$, corresponding to approximately half of a single oscillation cycle. Inverting the sign of the electric field of the pump pulse results in an opposite sign of changes in the diffraction intensity transients (Fig. 2B). Such behavior is expected when it is the electric field, and not simple heating, that drives the spin motion. When $\mathrm{TbMnO}_{3}$ is in the non-multiferroic SDW phase $(T=30 \mathrm{~K})$, the oscillation in the peak intensity following the pump is strongly suppressed (Fig. 2C). This temperature dependence gives strong evidence that the THz-induced spin motion is correlated with the presence of multiferroicity. At $30 \mathrm{~K}$ we tentatively attribute the slight drop of overall intensity after the pump to heating effects from absorption of the THz pulse, which leads to an estimated temperature increase of less than $0.05 \mathrm{~K}(34)$.

To better understand the time dependence of the spin response, we construct a very simple model of the system as two independent simple harmonic oscillators at the electromagnon resonance frequencies of $0.7 \mathrm{THz}$ and $1.8 \mathrm{THz}(34)$. Although not a perfect match to the data, the behavior of the conjugate momentum of the higher frequency oscillator successfully reproduces the general shape of the oscillation and the delay between the driving electric field and the changes in $\mathrm{x}$-ray diffraction (Fig. 2A and 2B). The agreement is much worse for either canonical coordinate of the lower frequency oscillator, suggesting that off-resonant excitation of the lower energy electromagnon or other purely magnetic modes is not consistent with the measured shape or delay of the response (34).

Resonant x-ray scattering at the $\mathrm{Mn} L$-edge is predominantly sensitive to the magnetic moment of the Mn $3 d$ shell (35). An analysis of how different spin motions contribute to the intensity of the diffraction peak allows us to test which of them are involved in the observed 
oscillations. We consider two components of the induced spin motion, motivated by the current understanding of spin dynamics in this system. In the first component the spins move in antiphase within the spin cycloid plane, the pattern widely considered to be responsible for the infrared activity of the $1.8 \mathrm{THz}$ electromagnon (28). The driving electric field applies an effective "force" to this component. In our model of the electromagnon as a harmonic oscillator, this component of the spin motion should then be identified with the "position" of the oscillator. In the proposed pure spin Hamiltonian for this system (18), the conjugate momentum must be a spin motion orthogonal to this position coordinate. Numerical simulations based on this Hamiltonian have predicted that a sufficiently intense $\mathrm{THz}$ pulse in resonance with the $1.8 \mathrm{THz}$ electromagnon can induce coherent rotation of the spin cycloid plane about the $b$ axis until it reaches another stable orientation in either the $(a b)$ or $(b c)$ plane (18). In our experiment the effective THz pulse field strength is over two orders of magnitude lower than used in these simulations (34) and so we do not expect to see a persistent domain reorientation. Instead, we propose to consider a smaller rotation of the spin cycloid plane about the $b$ axis as a second component of the spin motion that corresponds to the conjugate momentum for the $1.8 \mathrm{THz}$ resonance.

We model these two spin motion patterns separately as distortions to the equilibrium magnetic structure which influence the magnetic structure factor (34). We then calculate the intensity of the $(0 q 0)$ diffraction peak as a function of each coordinate of the spin motion (Fig. 3). For the in-plane motion the intensity of the diffraction peak is an even function of the spin coordinate, giving a decrease of the diffracted intensity with twice the frequency of the spin motion (Fig. 3A). For the spin cycloid plane rotation, the change of diffracted intensity is an odd function of the rotation angle. This motion then leads to a modulation of the diffraction intensity 
with the same frequency as the spin motion (Fig. 3B). For a field-driven excitation process we expect the spin motion frequency to be the same as the frequency of $\mathrm{THz}$ pump. We conclude that the main motion visible in our experiment is a rotation of the spin cycloid plane. The inplane spin motion may also be present, but its response would be suppressed at the current experimental time resolution. This is consistent with our harmonic oscillator model, which suggests that we see primarily dynamics of the conjugate momentum of the resonance.

For $\pi$-polarized x-rays at the $\mathrm{Mn} L_{2}$ edge the scattering intensity is a strongly varying function of the sample azimuth (rotation about the Bragg wave vector) (34). Rotation of the spin cycloid plane about the $b$ axis induced by the THz pulse is equivalent to rotating the sample about the $(0 q 0)$ scattering vector. Hence we interpret the data quantitatively by comparing the change of the intensity of the diffraction peak seen in the pump-probe trace with the change seen upon rotating the sample by a small angle around the azimuth of $45^{\circ}$ in equilibrium conditions (Fig. 4). We estimate that the observed $(1.35 \pm 0.12) \%$ maximum change of peak intensity corresponds to an amplitude of spin cycloid plane rotation equal to $(4.2 \pm 0.4)^{\circ}(34)$. We expect higher fields will lead to larger spin cycloid rotations. A simple linear extrapolation suggests that THz pulses with an amplitude of 1-2 MV/cm inside the sample could lead to spin cycloid rotations on the order of $90^{\circ}$. We can compare this against the model of Ref. (18), which predicts switching at $14-15 \mathrm{MV} / \mathrm{cm}$ for single-cycle $\mathrm{THz}$ pulses.

Our results show that intense $\mathrm{THz}$ pulses can modify the magnetic order in a multiferroic. Given that $\mathrm{TbMnO}_{3}$ is a model compound for a large group of materials with noncollinear spin order, our results serve as a proof-of-principle for a wide range of compounds. Moreover, the presence of magnetoelectric coupling in multiferroic heterostructures encourages a search for similar mechanisms as a basis for technologically feasible multiferroic devices. 


\section{References and Notes:}

1. E. Beaurepaire, J. C. Merle, A. Daunois, J. Y. Bigot, Ultrafast spin dynamics in ferromagnetic nickel. Phys. Rev. Lett. 76, 4250 (1996).

2. A. Kirilyuk, A. V. Kimel, T. Rasing, Ultrafast optical manipulation of magnetic order. Rev. Mod. Phys. 82, 2731 (2010).

3. T. Kampfrath et al., Coherent terahertz control of antiferromagnetic spin waves. Nature Photon. 5, 31 (2011).

4. K. Yamaguchi, M. Nakajima, T. Suemoto, Coherent control of spin precession motion with impulsive magnetic fields of half-cycle terahertz radiation. Phys. Rev. Lett. 105, 237201 (2010)

5. C. Vicario et al., Off-resonant magnetization dynamics phase-locked to an intense phasestable terahertz transient. Nature Photon. 7, 720 (2013).

6. W. Eerenstein, N. D. Mathur, J. F. Scott, Multiferroic and magnetoelectric materials. Nature 442, $759(2006)$

7. H. Katsura, N. Nagaosa, A. V. Balatsky, Spin current and magnetoelectric effect in noncollinear magnets. Phys. Rev. Lett. 95, 057205 (2005).

8. M. Mostovoy, Ferroelectricity in spiral magnets. Phys. Rev. Lett. 96, 067601 (2006).

9. S.-W. Cheong, M. Mostovoy, Multiferroics: a magnetic twist for ferroelectricity. Nature Mater. 6, 13 (2007).

10. T. Lottermoser et al., Magnetic phase control by an electric field. Nature 430, 541 (2004). 
11. Y. Bodenthin et al., Manipulating the magnetic structure with electric fields in multiferroic $\mathrm{ErMn}_{2} \mathrm{O}_{5}$. Phys. Rev. Lett. 100, 027201 (2008).

12. Y. Yamasaki et al., Electric control of spin helicity in a magnetic ferroelectric. Phys. Rev. Lett. 98, 147204 (2007).

13. Y. J. Choi, C. L. Zhang, N. Lee, S. W. Cheong, Cross-control of magnetization and polarization by electric and magnetic fields with competing multiferroic and weakferromagnetic phases. Phys. Rev. Lett. 105, 097201 (2010).

14. T. Hoffmann, P. Thielen, P. Becker, L. Bohatý, M. Fiebig, Time-resolved imaging of magnetoelectric switching in multiferroic $\mathrm{MnWO}_{4}$. Phys. Rev. B 84, 184404 (2011).

15. S. L. Johnson et al., Femtosecond dynamics of the collinear-to-spiral antiferromagnetic phase transition in CuO. Phys. Rev. Lett. 108, 037203 (2012).

16. I. P. Handayani et al., Dynamics of photo-excited electrons in magnetically ordered $\mathrm{TbMnO}_{3}$. J. Phys. Cond. Matter 25, 116007 (2013).

17. D. S. Rana et al., Understanding the nature of ultrafast polarization dynamics of ferroelectric memory in the multiferroic $\mathrm{BiFeO}_{3}$. Adv. Mater. 21, 2881 (2009).

18. M. Mochizuki, N. Nagaosa, Theoretically predicted picosecond optical switching of spin chirality in multiferroics. Phys. Rev. Lett. 105, 147202 (2010).

19. T. Kimura et al., Magnetic control of ferroelectric polarization. Nature 426, 55 (2003).

20. M. Kenzelmann et al., Magnetic inversion symmetry breaking and ferroelectricity in $\mathrm{TbMnO}_{3}$. Phys. Rev. Lett. 95, 087206 (2005). 
21. I. A. Sergienko, E. Dagotto, Role of the Dzyaloshinskii-Moriya interaction in multiferroic perovskites. Phys. Rev. B 73, 094434 (2006).

22. A. Malashevich, D. Vanderbilt, First principles study of improper ferroelectricity in $\mathrm{TbMnO}_{3}$. Phys. Rev. Lett. 101, 037210 (2008).

23. H. C. Walker et al., Femtoscale magnetically induced lattice distortions in multiferroic $\mathrm{TbMnO}_{3}$. Science 333, 1273 (2011).

24. S. B. Wilkins et al., Nature of the magnetic order and origin of induced ferroelectricity in $\mathrm{TbMnO}_{3}$. Phys. Rev. Lett. 103, 207602 (2009).

25. A. Pimenov et al., Possible evidence for electromagnons in multiferroic manganites. Nature Phys. 2, 97 (2006).

26. Y. Takahashi et al., Evidence for an electric-dipole active continuum band of spin excitations in multiferroic $\mathrm{TbMnO}_{3}$. Phys. Rev. Lett. 101, 187201 (2008).

27. A. Pimenov et al., Magnetic and magnetoelectric excitations in $\mathrm{TbMnO}_{3}$. Phys. Rev. Lett. 102, 107203 (2009).

28. R. Valdés Aguilar et al., Origin of electromagnon excitations in multiferroic $R \mathrm{MnO}_{3}$. Phys. Rev. Lett. 102, 047203 (2009).

29. P. Rovillain et al., Magnetic field induced dehybridization of the electromagnons in multiferroic $\mathrm{TbMnO}_{3}$. Phys. Rev. Lett. 107, 027202 (2011).

30. M. Mochizuki, N. Furukawa, N. Nagaosa, Theory of electromagnons in the multiferroic mn perovskites: the vital role of higher harmonic components of the spiral spin order. Phys. Rev. Lett. 104, 177206 (2010). 
31. H. Katsura, A. V. Balatsky, N. Nagaosa, Dynamical magnetoelectric coupling in helical magnets. Phys. Rev. Lett. 98, 027203 (2007).

32. A. M. Shuvaev, V. D. Travkin, V. Y. Ivanov, A. A. Mukhin, A. Pimenov, Evidence for electroactive excitation of the spin cycloid in $\mathrm{TbMnO}_{3}$. Phys. Rev. Lett. 104, 097202 (2010).

33. C. Ruchert, C. Vicario, C. P. Hauri, Spatiotemporal focusing dynamics of intense supercontinuum THz pulses. Phys. Rev. Lett. 110, 123902 (2013).

34. See Supporting Online Material.

35. S. W. Lovesey, S. P. Collins, X-ray Scattering and Absorption by Magnetic Materials. (Clarendon, Oxford 1996).

36. W. F. Schlotter et al., The soft x-ray instrument for materials studies at the linac coherent light source x-ray free-electron laser. Rev. Sci. Instrum. 83, 043107 (2012).

37. D. Doering et al., Development of a compact fast CCD camera and resonant soft x-ray scattering endstation for time-resolved pump-probe experiments. Rev. Sci. Instrum. 82, $073303(2011)$.

38. S. Lovesey et al., Melting of chiral order in terbium manganate $\left(\mathrm{TbMnO}_{3}\right)$ observed with resonant x-ray Bragg diffraction. J. Phys. Cond. Matter 25, 362202 (2013).

39. Z. Yang et al., Large-size bulk and thin-film stilbazolium-salt single crystals for nonlinear optics and THz generation. Adv. Funct. Mater. 17, 2018 (2007).

40. P. C. M. Planken, H.-K. Nienhuys, H. J. Bakker, T. Wenckebach, Measurement and calculation of the orientation dependence of terahertz pulse detection in ZnTe. J. Opt. Soc. Am. B 18, 313 (2001). 
41. M. C. Hoffmann, J. A. Fülöp, Intense ultrashort terahertz pulses: generation and applications. J. Phys. D: Appl. Phys. 44, 083001 (2011).

42. H. Hirori, A. Doi, F. Blanchard, K. Tanaka, Single-cycle terahertz pulses with amplitudes exceeding $1 \mathrm{MV} / \mathrm{cm}$ generated by optical rectification in $\mathrm{LiNbO}_{3}$. Appl. Phys. Lett. 98, $091106(2011)$.

43. N. Kida et al., Terahertz time-domain spectroscopy of electromagnons in multiferroic perovskite manganites. J. Opt. Soc. Am. B 26, A35 (2009).

44. M. Beye et al., X-ray pulse preserving single-shot optical cross-correlation method for improved experimental temporal resolution. Appl. Phys. Lett. 100, 121108 (2012).

45. J. M. Fornies-Marquina, J. Letosa, M. Garcia-Gracia, J. M. Artacho, Error propagation for the transformation of time domain into frequency domain. IEEE Trans. Magn. 33, 1456 (1997).

46. U. Staub et al., Polarization analysis in soft X-ray diffraction to study magnetic and orbital ordering. J. Synchrotron. Radiat. 15, 469 (2008).

47. J. P. Hill, D. F. McMorrow, Resonant exchange scattering: polarization dependence and correlation function. Acta Crystallogr. A52, 236 (1996).

Acknowledgments: This research was carried out on the SXR Instrument at the LCLS, a division of SLAC and an Office of Science user facility operated by Stanford University for the U.S. Department of Energy (DOE). The SXR Instrument is funded by a consortium including the LCLS, Stanford University through SIMES, LBNL, the University of Hamburg through the BMBF priority program FSP 301, and the Center for Free Electron Laser Science (CFEL). This 
research was supported by the NCCR MUST and NCCR MaNEP, funded by the Swiss National Science Foundation, and by the Swiss National Science Foundation (Grant No. 200021_144115). Our ultrafast activities are supported by the ETH Femtosecond and Attosecond Science and Technology (ETH-FAST) initiative as part of the NCCR MUST program. The Advanced Light Source is supported by DOE under contract No. DE-AC02-05CH1 1231. Crystal growth work at IQM was supported by DOE, Office of Basic Energy Sciences, Division of Materials Sciences and Engineering under Award DE-FG02-08ER46544. W.-S. L., Y.-D. C., and R. G. M. are supported by the Department of Energy, Office of Basic Energy Sciences, Materials Sciences and Engineering Division, under contract DE-AC02-76SF00515. S. L. J. and U. S. contributed equally to this work. 

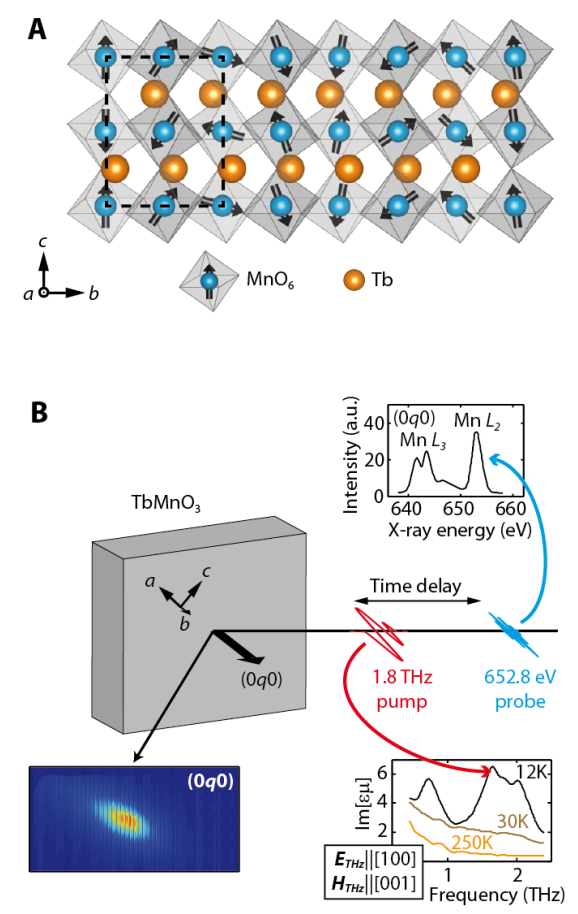

Fig. 1. Experimental setup. (A) The magnetic structure of $\mathrm{TbMnO}_{3}$ below $27 \mathrm{~K}$. The spins of the $\mathrm{Mn} 3 d$ shells (black arrows) form a cycloid propagating within the $(b c)$ crystallographic plane. The oxygens are represented by gray octahedra around the Mn atoms (blue spheres). The black dashed box indicates a unit cell. (B) Schematic of the experiment. A THz pulse resonant with the strongest electromagnon (lower right inset (26)) excites spin motion in the sample. An X-ray pulse resonant with the $\mathrm{Mn} L_{2}$ edge (upper inset) measures the response as changes in the intensity of the $(0 q 0)$ diffraction peak (lower left inset). 

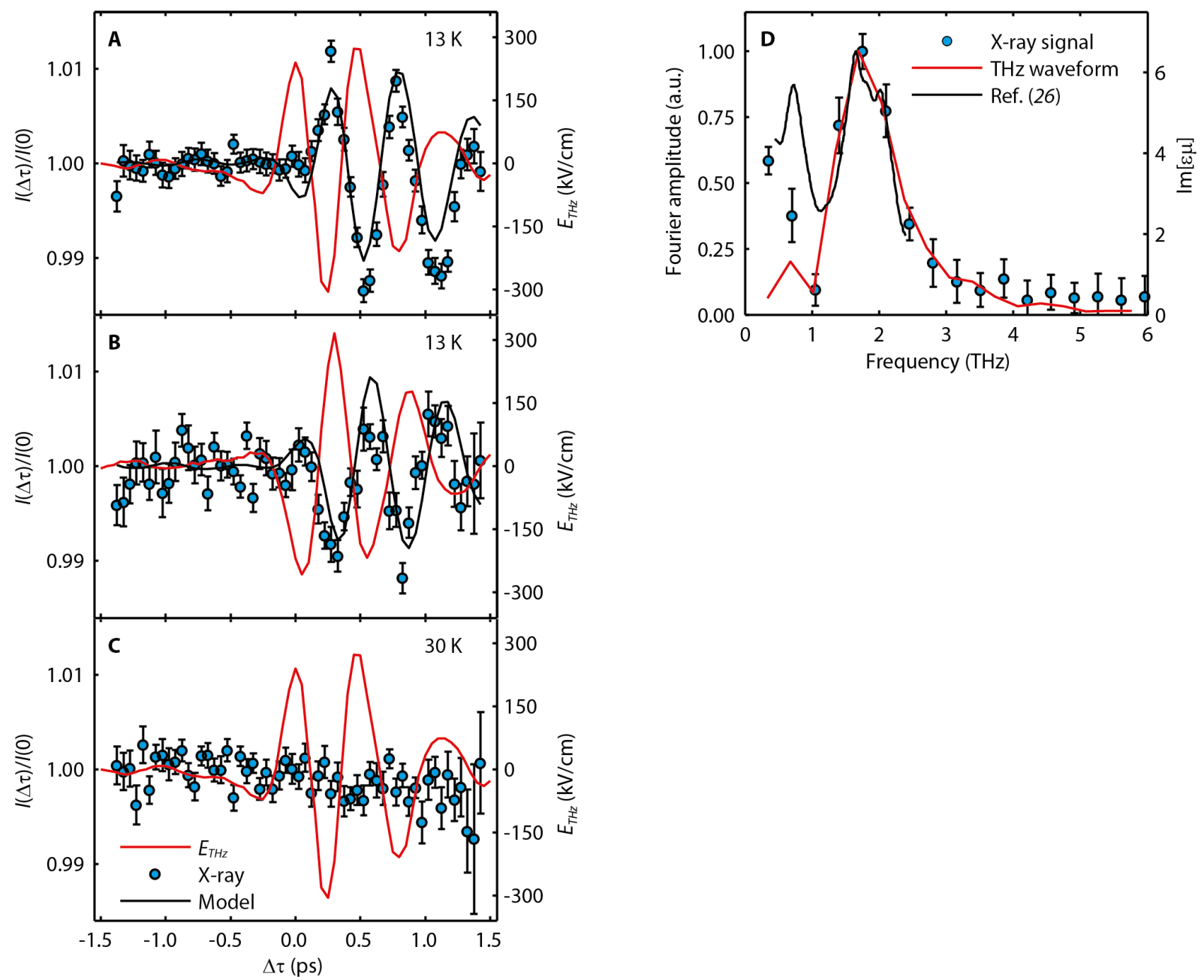

Fig. 2. Time-dependent behavior. The magnetic diffraction intensity of the $(0 q 0)$ peak of $\mathrm{TbMnO}_{3}$ (blue symbols, left axis), compared with the pump trace (red solid line, right axis) as a function of the time delay. (A, B) The response of the crystal in the multiferroic phase $(T=13 \mathrm{~K})$ for opposite signs of the driving electric field. The solid black lines are based on a model discussed in the text. (C) The response in the SDW phase $(T=30 \mathrm{~K})$. (D) Fourier transform of the $\mathrm{THz}$ and $\mathrm{x}$-ray traces from (A). 
A
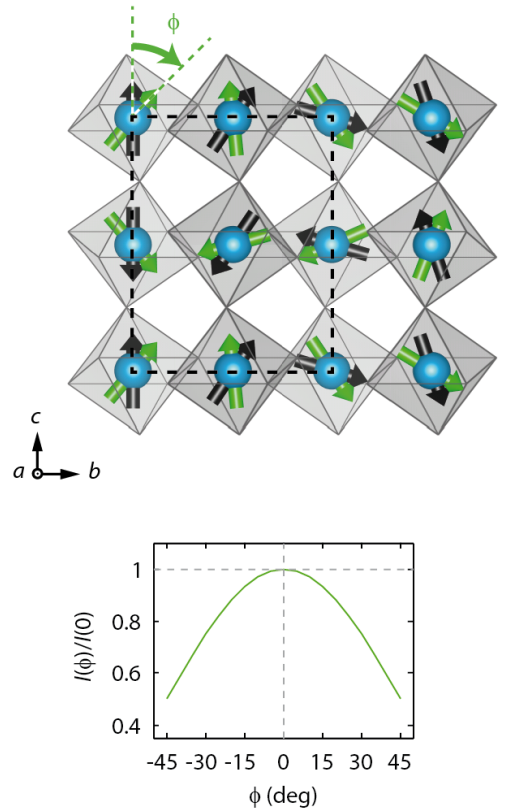

B
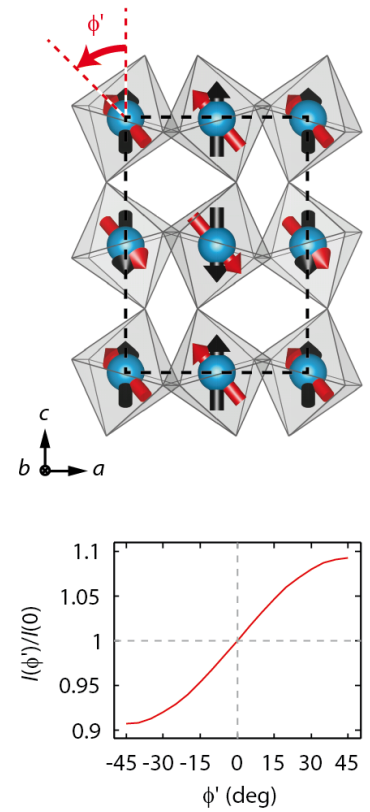

Fig. 3. Spin-motion patterns analyzed to interpret the time-dependent data. The upper panels illustrate the different patterns of how the magnetic structure changes. Black arrows denote how the spins are oriented in the ground state. Color arrows indicate the spin directions at one of the extremes of the excited motion. Tb ions have been removed for clarity. The lower panels show calculations of the changes in $(0 q 0)$ peak intensity as a function of the motion coordinate. (A) Antiphase oscillation within the spin cycloid plane, parameterized using the spin rotation coordinate $\varphi$ and viewed along the $a$ axis. (B) Coherent rotation of the spin cycloid plane by an angle $\varphi^{\prime}$ about the crystallographic $b$ axis viewed along the $b$ axis. 


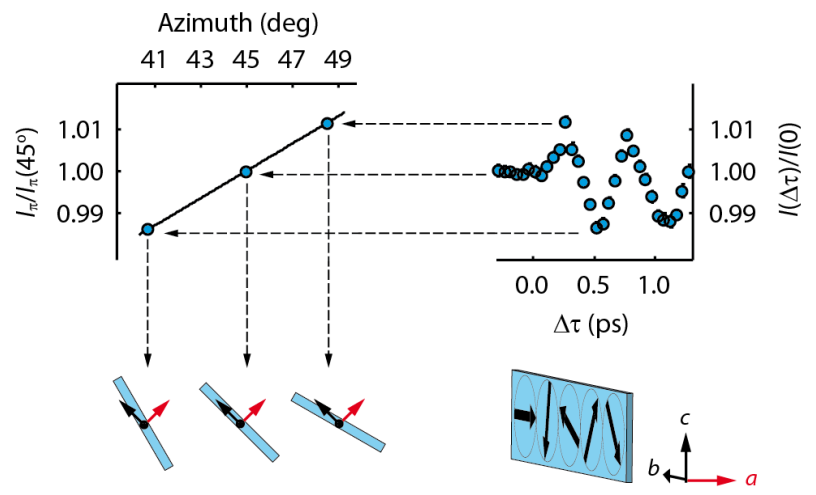

Fig. 4. The diffracted intensity vs. spin cycloid rotations. Left: Azimuthal dependence of the $(0 q 0)$ peak for the $\pi$-polarized incident $\mathrm{x}$-rays divided by the diffracted intensity at an azimuth of $45^{\circ}$. Right: Time-resolved diffracted intensity normalized to the intensity before excitation. The blue plane represents a plane of a single spin cycloid propagating along the crystallographic $b$ axis. The $a$ axis is marked with red. The angles of rotation in the drawing have been exaggerated for clarity.

\section{Supplementary Materials:}

Materials and Methods

Figures S1-S3

References (36-47) 


\section{Science \\ $\triangle 1$ AAAS}

\section{Supplementary Materials for}

Large Amplitude Spin Dynamics Driven by a THz Pulse in Resonance with an Electromagnon

T. Kubacka*, J.A. Johnson, M.C. Hoffmann, C. Vicario, S. de Jong, P. Beaud, S. Grübel, S-W. Huang, L. Huber, L. Patthey, Y-D. Chuang, J.J. Turner, G.L. Dakovski, W-S. Lee, M.P. Minitti, W. Schlotter, R. G. Moore, C.P. Hauri, S.M. Koohpayeh, V. Scagnoli, G. Ingold, S.L. Johnson, U. Staub

*correspondence to: tkubacka@phys.ethz.ch

\section{This PDF file includes:}

Materials and Methods

Supplementary Text

Figs. S1 to S3

References and Notes 


\section{Materials and Methods}

Sample preparation

A high quality, stoichiometric $\mathrm{TbMnO}_{3}$ single crystal was grown by the optical floating zone technique at the zoning rate of $0.5 \mathrm{~mm} / \mathrm{h}$ with rotation rate of $15 \mathrm{rpm}$ for the growing crystal and $0 \mathrm{rpm}$ for the feed rod under static argon. The crystal was oriented using Laue backscattering and cut to expose the (010) face with an approximately $3^{\circ}$ miscut. The surface of the sample was polished and afterwards the sample was annealed in air in $650^{\circ} \mathrm{C}$ for $110 \mathrm{~h}$. The dimensions of the crystal are approximately $2 \mathrm{~mm} \times 2 \mathrm{~mm}$ $\mathrm{x} 3 \mathrm{~mm}$.

The Pbnm orthorhombic convention is used to describe the crystal axes.

\section{Experimental setup}

The setup is sketched in the Fig. S1. The experiment was performed at the Soft XRay (SXR) beamline at the Linac Coherent Light Source X-ray Free Electron Laser (LCLS FEL) (36), using the Resonant Soft X-ray Scattering endstation (37). The diffraction peak was measured using the fast-CCD camera (fCCD) (37).

The FEL was operated at a repetition rate of $60 \mathrm{~Hz}$. The x-ray absorption spectrum of $\mathrm{TbMnO}_{3}$ at $15 \mathrm{~K}$ was measured around the $\mathrm{Mn} L_{2}$ edge in order to calibrate the incident $\mathrm{x}$-ray energy. The $(0 q 0)$ peak was measured in the in-plane scattering geometry using $\pi$-polarized x-ray pulses with duration of $100 \mathrm{fs}$ FWHM. Previous work on this system under equilibrium conditions has shown using $\mathrm{x}$-ray polarization analysis that the dominant scattering contribution for this diffraction peak is magnetic in origin (38). Kirkpatrick-Baez optics were used to focus the beam to a spot size with a diameter of 300 $\mu \mathrm{m}$.

We used a high power Ti:Sapphire laser synchronized with the FEL trigger to produce $120-\mathrm{fs}$ long pulses with a center wavelength of $800 \mathrm{~nm}$. The main beam was split into three branches: (1) a branch leading to the high-power Optical Parametric Amplifier (OPA), used later to generate the THz pulses, (2) a branch hitting the sample collinearly with the x-rays, used for alignment and timing purposes, and (3) a branch used to determine the mutual time jitter of the x-ray and laser pulses. The $\mathrm{x}$-rays, $800 \mathrm{~nm}$ beam and the $\mathrm{THz}$ beam entered the sample collinearly.

\section{$\underline{\mathrm{THz}}$ generation and characterization}

The phase-stable, THz pulses were generated using the organic crystal 4-N,Ndimethylamino-4'-N'-methyl-stilbazolium 2,4,6-trimethylbenzenesulfonate (DSTMS) (39), with a diameter of $6 \mathrm{~mm}$ and thickness of $0.59 \mathrm{~mm}$. The DSTMS crystal was pumped with IR pulses with a center wavelength of $1500 \mathrm{~nm}$ and a duration of approximately $120 \mathrm{fs}$ FWHM. The THz pulses were polarized horizontally and hit the sample with p-polarization. Inverting the sign of the $\mathrm{THz}$ waveform was achieved by rotating the DSTMS crystal by $180^{\circ}$ around the beam propagation direction. The $\mathrm{THz}$ beam was focused on the sample using an off-axis parabolic mirror placed inside the RSXS chamber. The THz spot size at focus had a $1 / \mathrm{e}^{2}$ diameter of approximately 750 $\mu \mathrm{m}$.

The shape of the $\mathrm{THz}$ waveform was measured using electro-optical sampling (40) on the optimally oriented $150 \mu \mathrm{m}$ thick GaP crystal on $2 \mathrm{~mm}$ thick GaP substrate, placed 
in the position of the $\mathrm{TbMnO}_{3}$ sample. The maximal modulation of approximately $45 \%$ of the electro-optical signal measured with the balanced photodiode corresponds to an amplitude of the electric field of the THz pulse of $300 \mathrm{kV} / \mathrm{cm}$, assuming an electro-optic coefficient of GaP $r_{41}=0.88 \mathrm{pm} / \mathrm{V}$ and refractive indices of $\mathrm{GaP} n_{800 \mathrm{~nm}}=3.2, n_{T H z}=3.34$ $(41,42)$. Although the absolute sign of the electric field is ambiguous in our measurements, relative changes in the sign of the field on rotating the DSTMS crystal by $180^{\circ}$ are well characterized.

Because of the Bragg scattering geometry, the $\mathrm{THz}$ beam was incident on the surface of $\mathrm{TbMnO}_{3}$ at an angle of approximately $63^{\circ}$. The amplitude of the component of the electric field of the THz along the $a$ axis inside the sample is estimated to be 65 $\mathrm{kV} / \mathrm{cm}$, using $E_{\| a}=1 / \sqrt{2} \cdot t_{p}\left(\theta_{\text {inc }}\right) \cdot \cos \left(\theta_{t r}\right) \cdot E$, where $t_{p}=0.31$ is the Fresnel transmission coefficient for the p-polarized field with amplitude $E$ at the incident angle of $\theta_{i n c}=63^{\circ}$, and $\theta_{t r}$ is the angle between the direction of propagation of the refracted beam and the surface normal. In these calculations we use only the real part of refractive index of $\mathrm{TbMnO}_{3}$ at $1.8 \mathrm{THz}, n=4.31$, assuming it is equal along $a$ and $c$ axes $(26,43)$.

The temperature rise of the sample in the sinusoidal phase is calculated assuming that all the energy of the incident $\mathrm{THz}$ pulse is absorbed by the sample and converted to heat. We assume that all the incident $\mathrm{THz}$ radiation is absorbed $\left(T_{p}\left(63^{\circ}\right)=0.89\right)$ and that the heat capacity of $\mathrm{TbMnO}_{3}$ equals to $14 \mathrm{Jmol}^{-1} \mathrm{~K}^{-1}$ at $30 \mathrm{~K}(19)$. The $\mathrm{THz}$ penetration depth in the SDW phase is estimated to be approximately $60 \mu \mathrm{m}$ at $1.8 \mathrm{THz}$, assuming that the real part of the refractive index is approximately the same in the multiferroic and the SDW phase $(26,43)$.

\section{Overlap and timing}

The $\mathrm{x}$-ray penetration depth $(0.06 \mu \mathrm{m}$ at $652.8 \mathrm{eV})$ is much smaller than the $\mathrm{THz}$ penetration depth (approximately $15 \mu \mathrm{m}$ at $1.8 \mathrm{THz}$ in the multiferroic phase of $\mathrm{TbMnO}_{3}$ calculated using the imaginary part of the refractive index (43)). Spatial overlap between the $\mathrm{x}$-ray and $800 \mathrm{~nm}$ beams was achieved imaging the $\mathrm{x}$-ray induced fluorescence from a YAG crystal moved to the sample position. The time overlap between the $\mathrm{x}$-ray and $\mathrm{THz}$ pulses was obtained in a two-step process using an intermediate overlap with the $800 \mathrm{~nm}$ beam. First, we found overlap in time between the x-ray and $800 \mathrm{~nm}$ pulses in an X-ray pump, $800 \mathrm{~nm}$ probe experiment using $500 \mu \mathrm{m}$ thick ZnTe crystal placed in the position of the $\mathrm{TbMnO}_{3}$ sample. Soft x-rays excite carriers in $\mathrm{ZnTe}$, inducing a sudden drop in transmittivity of the optical beam, which allows determination of time zero. Afterwards, spatial and temporal overlap between the $800 \mathrm{~nm}$ beam and the THz were established using electro-optical sampling on a GaP crystal moved to the sample position. We estimate the accuracy of time zero determination to be 25 fs from uncertainties in the measurement, assuming that the optical response of ZnTe to x-ray excitation is an ideal step function in the limit of zero pulse duration.

The delay time between the $\mathrm{x}$-rays and the $\mathrm{THz}$ was changed using a continuously moving encoded mechanical delay stage put in the branch of $1500 \mathrm{~nm}$ beam (DL1 on Fig. $\mathrm{S} 1)$. For every FEL shot, the jitter between the x-ray pulse and the main $800 \mathrm{~nm}$ pulse was monitored using a time-to-space mapping cross-correlator (44), resulting in an effective time resolution of approximately 250 fs FWHM. 


\section{Data collection and analysis}

For each shot we extract the intensity of the diffraction peak, the time delay between the pump and the probe and the intensity $I_{0}$ of the incident x-ray beam. The intensity of the diffraction peak is calculated by integration within a set region of interest on the image from the fCCD. For each shot the image is corrected for the inherent background of the camera by subtracting the background frame recorded before every data run, and for the short-time-scale drift of the overall readout from the camera by subtracting the intensity integrated within the region of interest placed far away on the detector from the diffraction peak. The relative incident $\mathrm{x}$-ray pulse intensity is determined for each shot by measuring the photoelectron current from an aluminum-coated foil placed in the beamline (36).

Because the optical delay stage was scanned continuously, the total number of shots contributing to a single data point is different. For data in Fig. 2A the total number of shots per data point around time zero is approximately 12 000, while for data in Fig. 2B$\mathrm{C}$ it is approximately 5000 . We cut off the delay time at the bin where the number of shots per data point drops to half of the value around time zero.

To account for large fluctuations of the x-ray pulse intensity, we use a weighted average to obtain diffraction intensity for one bin for every data run, where we weight the diffraction intensity by the corresponding value of the $I_{0}$ for all shots. To correct for nonlinearities in $I_{0}$ readout we use a calibration curve, created by plotting the diffraction intensity as a function of $I_{0}$ and fitting it with a second order polynomial. We assume that the diffraction efficiency of the sample is a function well-described by simply a fraction of incident $\mathrm{x}$-ray intensity and that the readout from the fCCD detector is linear as a function of intensity of the diffraction peak, which is well supported by the fact that the shape of the diffraction peak on the detector barely changes as a function of the x-ray intensity.

In order to calculate the error bars of the pump-probe traces, we split the data runs contributing to a single measurement into slices consisting of approximately 10000 shots each. For every slice, we extract a pump-probe trace by time-binning the data in 50-fs wide bins. Because the diffraction efficiency of the sample changes with time, we normalize the trace from every slice to the value of the signal before time zero. The traces from separate slices are then averaged to obtain the final pump-probe trace and the error bars for every bin are calculated as a standard error within each bin. For data in Fig. 2A, $2 \mathrm{~B}$ and $2 \mathrm{C}$ the number of slices was approximately 70,20 and 30 respectively.

The uncertainties for the Fourier-transformed spectra are calculated following (45).

\section{Supplementary Text}

Modeling the material response with a harmonic oscillator model

We model the system as a collection of two types of uncoupled, damped harmonic oscillators. The response in this region of the electromagnetic spectrum is dominated by two types of resonators with frequencies near $0.75 \mathrm{THz}$ and $1.8 \mathrm{THz}$. Let $q_{1}$ and $p_{1}$ be the "position" and "momentum" canonical coordinates for the lower energy oscillator. Similarly, let $q_{2}$ and $p_{2}$ be the canonical coordinates for the higher energy oscillator. The full dynamics are then given by a system of four first order ordinary differential equations 


$$
\begin{gathered}
\frac{d q_{j}}{d t}=p_{j} \\
\frac{d p_{j}}{d t}=f_{j}(t)-4 \pi \zeta_{j} v_{j} p_{j}-4 \pi^{2} v_{j}^{2} q_{j}
\end{gathered}
$$

where $v_{j}$ and $\zeta_{j}$ are the natural frequency and damping for each oscillator, and $f_{j}(t)$ is the driving force which we assume here is proportional to the electric field of the applied $\mathrm{THz}$ pulse. The solutions in the Fourier domain are

$$
\begin{aligned}
Q_{j}(v) & =\frac{F_{j}(v)}{4 \pi^{2}\left(v_{j}^{2}-v^{2}-2 i \zeta_{j} v_{j} v\right)} \\
P_{j}(v) & =\frac{i v F_{j}(v)}{2 \pi\left(v_{j}^{2}-v^{2}-2 i \zeta_{j} v_{j} v\right)}
\end{aligned}
$$

where $Q_{j}, P_{j}$ and $F_{j}$ denote the Fourier transforms of $q_{j}, p_{j}$ and $f_{j}$. Since the imaginary part of the dielectric permittivity $\varepsilon$ is proportional to a weighted sum of the imaginary parts of $Q_{1}(v)$ and $Q_{2}(v)$, we can use the published permittivity data (26) to estimate $v_{1}=0.75$ $\mathrm{THz}, v_{2}=1.85 \mathrm{THz}, \zeta_{1}=0.34$ and $\zeta_{2}=0.198$. The resulting fit is shown in Fig. S2A.

Upon inverse Fourier transform, we can then compare our model, one mode at a time, with the recorded time-domain material response. In Figs. S2B and S2C we plot both $q_{j}(t)$ and $p_{j}(t)$ for each oscillator. These curves are each scaled by a constant, adjusted individually to best match the data. For $p_{1}$ and $p_{2}$ the scaling constants are negative, an allowance for the ambiguity in the absolute sign of the effective force on the oscillator. These scaling factors are the only adjustable fit parameters in the model. As seen in Fig. S2B, $p_{2}(t)$ fits the data reasonably well, recovering the correct phase delay between the pump and observed x-ray oscillation. Neither $q_{1}(t)$ nor $p_{1}(t)$ reproduce the experimental data well, both reacting significantly earlier in time than the measured spin response. The "delay" with response to the driving field observed in the data is in fact a direct consequence of driving the oscillator in resonance. In resonance, energy is efficiently transferred to the oscillator and stored there until the natural damping effects lead to a decay of the coherent response. The fact that we observe this delay is also evidence that the observed dynamics are not related to the non-resonant excitation of other, purely magnetic resonances in system that we have not considered here.

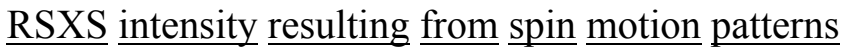

The azimuthal scans of the $(0 q 0)$ peak at the $\mathrm{Mn} L_{2}$ edge were measured in a separate experiment at the RESOXS endstation at the SIM beamline (Swiss Light Source, PSI) (46) for $\pi$ - and $\sigma$-polarized incident $x$-rays without the analysis of the diffracted beam polarization (Fig. S3) (38). The calculation of the Fourier coefficients of the magnetic structure of $\mathrm{TbMnO}_{3}$ in the multiferroic phase contributing to the $(0 q 0)$ peak visible in the resonant scattering experiment is performed using the program BasIreps 4.10 included in the FULLPROF suite. In the multiferroic phase two irreducible representations are required to describe the magnetic structure (20). The values of the components of the basis functions along the $b$ and $c$ axes are obtained from the fit to the experimental data (solid lines in Fig. S3). For the magnetic structure factor of a form $\mathbf{f}_{0 q 0}^{0}=(0, v, w)$ we obtain $v=0.57 \mathrm{i}$ and $w=1$. The directions and amplitudes of the contributions of magnetic moments visible in the experiment are then calculated from the 
Fourier coefficients. Our data are consistent with the cycloid propagating along the $b$ axis, however the Mn spin components along the $c$ axis we observe at this particular reflection are ordered ferromagnetically along the $c$ axis. The resonant x-ray diffracted intensity and the shape of the azimuthal scans are calculated numerically following Ref. (47) confirming a good agreement with the experimental data.

In the next step, the magnetic structure is deformed by rotating the spins on particular sites by an angle $\pm \varphi\left( \pm \varphi^{\prime}\right)$, in order to reproduce the magnetic structure during the spin excitation in a state "frozen" in time. For every $\varphi(\varphi$ '), we compute the magnetic structure factor of the resulting distorted magnetic structure. The intensity of the $(0 q 0)$ diffraction peak at azimuth of $45^{\circ}$ and $\pi$-polarized incident $\mathrm{x}$-rays is then calculated and normalized to the value obtained for $\varphi=0\left(\varphi^{\prime}=0\right)$ (the case of non-distorted cycloid).

Estimate of magnetic-field induced dynamics

To estimate the maximal effect of the non-resonant oscillating magnetic field component of the $\mathrm{THz}$ excitation on the observed $\mathrm{x}$-ray diffraction, we consider the unconstrained precession of the spin magnetic moments $\mathbf{S}_{\mathrm{i}}$ given by

$$
\frac{d \mathbf{S}_{\mathbf{i}}}{d t}=-\gamma \mathbf{S}_{\mathbf{i}} \times \mathbf{B}(t)
$$

where $\mathbf{B}(t)=\mathbf{B}_{0} g(t)$ is the applied magnetic field, $\gamma=1.76 \cdot 10^{11} \mathrm{rad} / \mathrm{T} \mathrm{s}$ is the gyromagnetic ratio, $\mathbf{B}_{0}$ is the magnitude and direction of the magnetic field, and $g(t)$ gives its time dependence. Assuming the changes in spin direction are small, to leading order we integrate to obtain

$$
\mathbf{S}_{\mathbf{i}}(t) \approx \mathbf{S}_{\mathbf{i}}^{\mathbf{0}}-\gamma \mathbf{S}_{\mathbf{i}}^{\mathbf{0}} \times \mathbf{B}_{\mathbf{0}} \int_{t_{s}}^{t} g\left(t^{\prime}\right) d t^{\prime}
$$

where $\mathbf{S}_{\mathrm{i}}{ }^{0}$ is the initial spin orientation at a time $t_{\mathrm{s}}$ well before the THz pulse interacts with the system. Since this relation is linear in $\mathbf{S}_{\mathrm{i}}{ }^{0}$ and the Fourier transform of the spin density is also a linear operation, the time-dependent magnetic structure factor $\mathbf{f}_{0 q 0}(t)$ has the same form

$$
\mathbf{f}_{0 q 0}(t) \approx \mathbf{f}_{0 q 0}^{0}-\gamma \mathbf{f}_{0 q 0}^{0} \times \mathbf{B}_{0} \int_{t_{s}}^{t} g\left(t^{\prime}\right) d t^{\prime}
$$

Using the value of the magnetic structure factor $\mathbf{f}_{0 q 0}^{0}$ inferred from the equilibrium measurements, we then calculate the effect on the diffracted intensity (47). For the $0.1 \mathrm{~T}$ peak fields used in our experiment we estimate a maximum change in the diffracted intensity of $0.035 \%$, well below the modulations we observe. We also note that the integral term in the time dependence would lead to a phase lag of $90^{\circ}$ in the response, which is also counter to observation. 


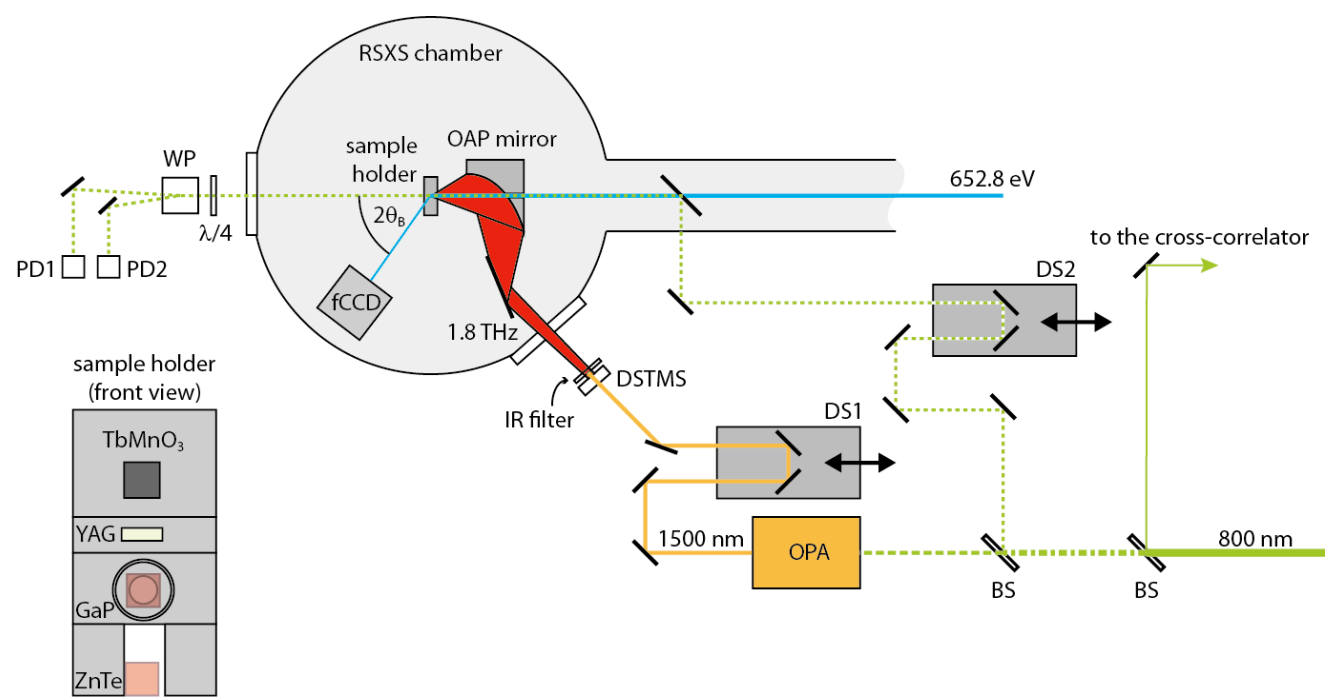

Fig. S1.

Sketch of the experimental setup. BS - beam splitter, DS - delay stage, OAP mirror off-axis parabolic mirror, PD - photodiode, WP - Wollaston prism, IR filter - low-pass filter made from $2 \mathrm{~mm}$ thick black polypropylene. For the $\mathrm{TbMnO}_{3}$ measurement, the sample holder was rotated to fulfill the Bragg scattering condition, for the electro-optical sampling and time zero determination the sample holder was rotated to achieve normal incidence of the beam. 

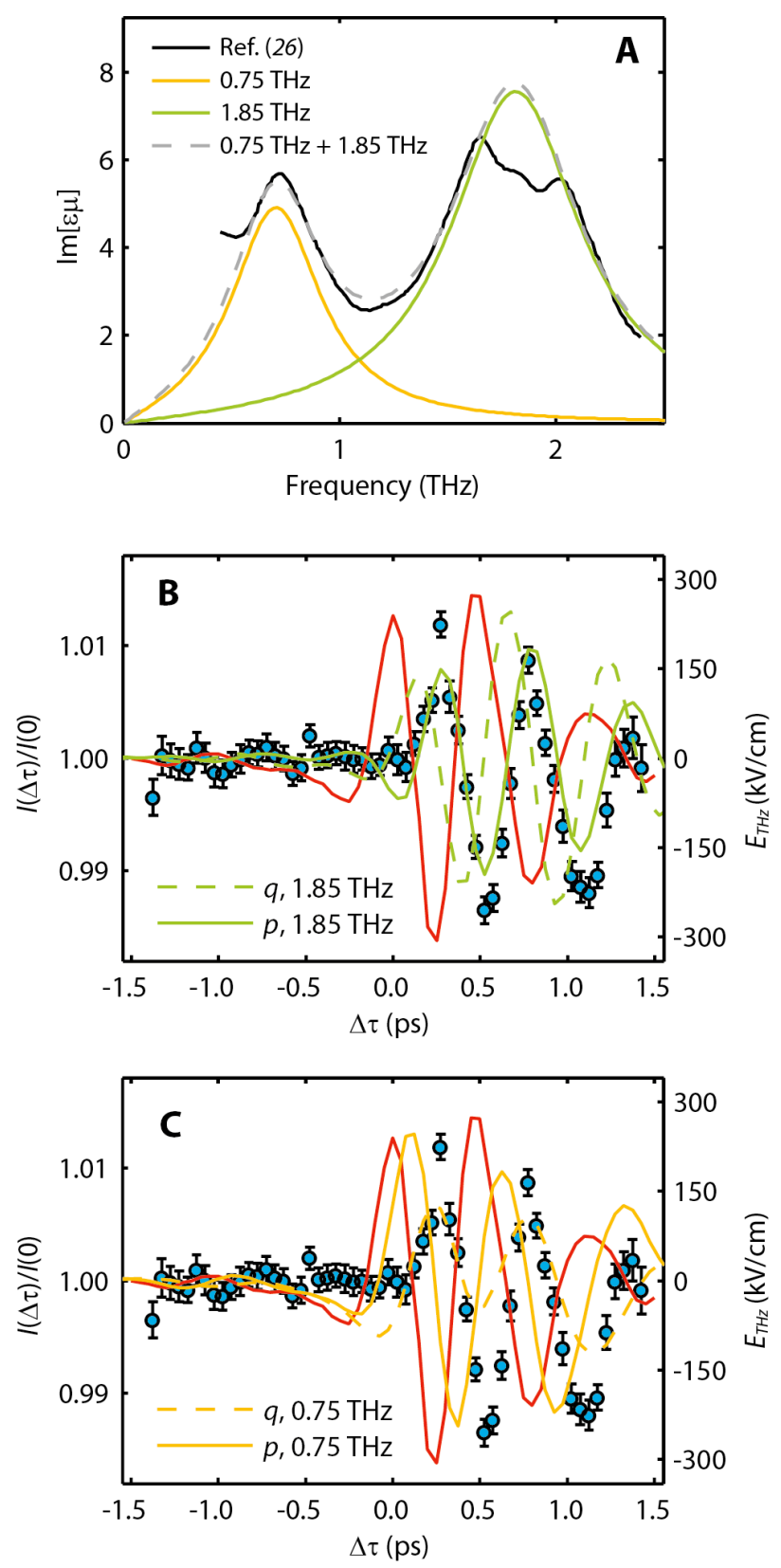

Fig. S2

(A) Measured $\operatorname{Im}[\varepsilon \mu]$ from ref. (26) compared to the fitted results from the model discussed in the text. (B) The simulated responses of the $1.85 \mathrm{THz}$ mode. (C) The simulated responses of the $0.75 \mathrm{THz}$ mode. In each plot red is the electro-optic $\mathrm{THz}$ trace, blue circles indicate the measured response, and the calculated responses $q_{\mathrm{j}}(\mathrm{t})$ and $p_{\mathrm{j}}(\mathrm{t})$ are shown as dashed and solid lines, respectively. The amplitudes of the simulated responses are individually scaled to best match the experimental data. 


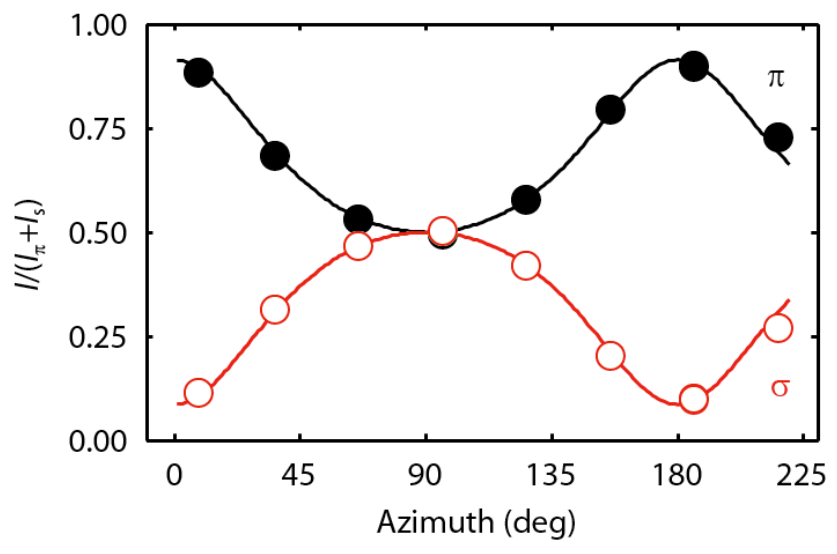

Fig. S3

Dependence of the $(0 q 0)$ diffraction peak intensity on the azimuthal angle for the $\pi$ - and $\sigma$-polarized incident x-rays (filled black and open red symbols, respectively), without outgoing x-ray polarization analysis. The azimuthal angle is defined to be zero when the $a$ axis is in the horizontal plane. Solid lines show the fit to the data. The uncertainties are smaller than the symbol size. 


\section{References and Notes:}

1. E. Beaurepaire, J. C. Merle, A. Daunois, J. Y. Bigot, Ultrafast spin dynamics in ferromagnetic nickel. Phys. Rev. Lett. 76, 4250 (1996).

2. A. Kirilyuk, A. V. Kimel, T. Rasing, Ultrafast optical manipulation of magnetic order. Rev. Mod. Phys. 82, 2731 (2010).

3. T. Kampfrath et al., Coherent terahertz control of antiferromagnetic spin waves. Nature Photon. 5, 31 (2011).

4. K. Yamaguchi, M. Nakajima, T. Suemoto, Coherent control of spin precession motion with impulsive magnetic fields of half-cycle terahertz radiation. Phys. Rev. Lett. 105, 237201 (2010).

5. C. Vicario et al., Off-resonant magnetization dynamics phase-locked to an intense phase-stable terahertz transient. Nature Photon. 7, 720 (2013).

6. W. Eerenstein, N. D. Mathur, J. F. Scott, Multiferroic and magnetoelectric materials. Nature 442, 759 (2006).

7. H. Katsura, N. Nagaosa, A. V. Balatsky, Spin current and magnetoelectric effect in noncollinear magnets. Phys. Rev. Lett. 95, 057205 (2005).

8. M. Mostovoy, Ferroelectricity in spiral magnets. Phys. Rev. Lett. 96, 067601 (2006).

9. S.-W. Cheong, M. Mostovoy, Multiferroics: a magnetic twist for ferroelectricity. Nature Mater. 6, 13 (2007).

10. T. Lottermoser et al., Magnetic phase control by an electric field. Nature 430, 541 (2004).

11. Y. Bodenthin et al., Manipulating the magnetic structure with electric fields in multiferroic $\mathrm{ErMn}_{2} \mathrm{O}_{5}$. Phys. Rev. Lett. 100, 027201 (2008).

12. Y. Yamasaki et al., Electric control of spin helicity in a magnetic ferroelectric. Phys. Rev. Lett. 98, 147204 (2007).

13. Y. J. Choi, C. L. Zhang, N. Lee, S. W. Cheong, Cross-control of magnetization and polarization by electric and magnetic fields with competing multiferroic and weakferromagnetic phases. Phys. Rev. Lett. 105, 097201 (2010).

14. T. Hoffmann, P. Thielen, P. Becker, L. Bohatý, M. Fiebig, Time-resolved imaging of magnetoelectric switching in multiferroic $\mathrm{MnWO}_{4}$. Phys. Rev. B 84, 184404 (2011).

15. S. L. Johnson et al., Femtosecond dynamics of the collinear-to-spiral antiferromagnetic phase transition in CuO. Phys. Rev. Lett. 108, 037203 (2012).

16. I. P. Handayani et al., Dynamics of photo-excited electrons in magnetically ordered $\mathrm{TbMnO}_{3}$. J. Phys. Cond. Matter 25, 116007 (2013).

17. D. S. Rana et al., Understanding the nature of ultrafast polarization dynamics of ferroelectric memory in the multiferroic $\mathrm{BiFeO}_{3}$. Adv. Mater. 21, 2881 (2009).

18. M. Mochizuki, N. Nagaosa, Theoretically predicted picosecond optical switching of spin chirality in multiferroics. Phys. Rev. Lett. 105, 147202 (2010). 
19. T. Kimura et al., Magnetic control of ferroelectric polarization. Nature 426, 55 (2003).

20. M. Kenzelmann et al., Magnetic inversion symmetry breaking and ferroelectricity in $\mathrm{TbMnO}_{3}$. Phys. Rev. Lett. 95, 087206 (2005).

21. I. A. Sergienko, E. Dagotto, Role of the Dzyaloshinskii-Moriya interaction in multiferroic perovskites. Phys. Rev. B 73, 094434 (2006).

22. A. Malashevich, D. Vanderbilt, First principles study of improper ferroelectricity in $\mathrm{TbMnO}_{3}$. Phys. Rev. Lett. 101, 037210 (2008).

23. H. C. Walker et al., Femtoscale magnetically induced lattice distortions in multiferroic $\mathrm{TbMnO}_{3}$. Science 333, 1273 (2011).

24. S. B. Wilkins et al., Nature of the magnetic order and origin of induced ferroelectricity in $\mathrm{TbMnO}_{3}$. Phys. Rev. Lett. 103, 207602 (2009).

25. A. Pimenov et al., Possible evidence for electromagnons in multiferroic manganites. Nature Phys. 2, 97 (2006).

26. Y. Takahashi et al., Evidence for an electric-dipole active continuum band of spin excitations in multiferroic $\mathrm{TbMnO}_{3}$. Phys. Rev. Lett. 101, 187201 (2008).

27. A. Pimenov et al., Magnetic and magnetoelectric excitations in $\mathrm{TbMnO}_{3}$. Phys. Rev. Lett. 102, 107203 (2009).

28. R. Valdés Aguilar et al., Origin of electromagnon excitations in multiferroic $R \mathrm{MnO}_{3}$. Phys. Rev. Lett. 102, 047203 (2009).

29. P. Rovillain et al., Magnetic field induced dehybridization of the electromagnons in multiferroic $\mathrm{TbMnO}_{3}$. Phys. Rev. Lett. 107, 027202 (2011).

30. M. Mochizuki, N. Furukawa, N. Nagaosa, Theory of electromagnons in the multiferroic mn perovskites: the vital role of higher harmonic components of the spiral spin order. Phys. Rev. Lett. 104, 177206 (2010).

31. H. Katsura, A. V. Balatsky, N. Nagaosa, Dynamical magnetoelectric coupling in helical magnets. Phys. Rev. Lett. 98, 027203 (2007).

32. A. M. Shuvaev, V. D. Travkin, V. Y. Ivanov, A. A. Mukhin, A. Pimenov, Evidence for electroactive excitation of the spin cycloid in $\mathrm{TbMnO}_{3}$. Phys. Rev. Lett. 104, 097202 (2010).

33. C. Ruchert, C. Vicario, C. P. Hauri, Spatiotemporal focusing dynamics of intense supercontinuum THz pulses. Phys. Rev. Lett. 110, 123902 (2013).

34. See Supporting Online Material.

35. S. W. Lovesey, S. P. Collins, X-ray Scattering and Absorption by Magnetic Materials. (Clarendon, Oxford 1996).

36. W. F. Schlotter et al., The soft x-ray instrument for materials studies at the linac coherent light source x-ray free-electron laser. Rev. Sci. Instrum. 83, 043107 (2012). 
37. D. Doering et al., Development of a compact fast CCD camera and resonant soft Xray scattering endstation for time-resolved pump-probe experiments. Rev. Sci. Instrum. 82, 073303 (2011).

38. S. Lovesey et al., Melting of chiral order in terbium manganate $\left(\mathrm{TbMnO}_{3}\right)$ observed with resonant x-ray Bragg diffraction. J. Phys. Cond. Matter 25, 362202 (2013).

39. Z. Yang et al., Large-size bulk and thin-film stilbazolium-salt single crystals for nonlinear optics and THz generation. Adv. Funct. Mater. 17, 2018 (2007).

40. P. C. M. Planken, H.-K. Nienhuys, H. J. Bakker, T. Wenckebach, Measurement and calculation of the orientation dependence of terahertz pulse detection in ZnTe. J. Opt. Soc. Am. B 18, 313 (2001).

41. M. C. Hoffmann, J. A. Fülöp, Intense ultrashort terahertz pulses: generation and applications. J. Phys. D: Appl. Phys. 44, 083001 (2011).

42. H. Hirori, A. Doi, F. Blanchard, K. Tanaka, Single-cycle terahertz pulses with amplitudes exceeding $1 \mathrm{MV} / \mathrm{cm}$ generated by optical rectification in $\mathrm{LiNbO}_{3}$. Appl. Phys. Lett. 98, 091106 (2011).

43. N. Kida et al., Terahertz time-domain spectroscopy of electromagnons in multiferroic perovskite manganites. J. Opt. Soc. Am. B 26, A35 (2009).

44. M. Beye et al., X-ray pulse preserving single-shot optical cross-correlation method for improved experimental temporal resolution. Appl. Phys. Lett. 100, 121108 (2012).

45. J. M. Fornies-Marquina, J. Letosa, M. Garcia-Gracia, J. M. Artacho, Error propagation for the transformation of time domain into frequency domain. IEEE Trans. Magn. 33, 1456 (1997).

46. U. Staub et al., Polarization analysis in soft X-ray diffraction to study magnetic and orbital ordering. J. Synchrotron. Radiat. 15, 469 (2008).

47. J. P. Hill, D. F. McMorrow, Resonant exchange scattering: polarization dependence and correlation function. Acta Crystallogr. A52, 236 (1996). 